\title{
Analysis of the Big Data Methods, Challenges and Applications in Intelligent Transportation Systems
}

\author{
MAHENDRA.G' ${ }^{1}$ ROOPASHREE.H. R' ${ }^{2}$ YOGEESH.A.C ${ }^{3}$ \\ ${ }^{1}$ Research Scholar, Department of CSE, GSSSIETW, Mysuru, VTU, Bela gavi, gg.mahendra@ gmail.com \\ ${ }^{2}$ Associa te Professor, Department of CSE, GSSSIETW, My suru, VTU, Bela gavi, roopashreehr@ gsss.edu.in \\ ${ }^{3}$ Assistant Professor, Department of CSE, Govt. Engineering College, Ku shalnagar, yogeesh13@ gmail.com
}

\begin{abstract}
Big Data is data that grows beyond the capability of traditional data mining methods. A big voluminous, heterogeneous, and speediness of data generated from numerous sensors attached near roadside infrastructure and devices a ssociated with the vehicles. We need to design Big data models for highly scalable, techniques for complex data types, robust to noise, insensitive to the input order, ability to handling high-dimensional data, interpretability, and usability. There are many challenges in enomous amount of data to stockpile, processing, and operate. Apache Spark, Hadoop, and Flink are the platforms of Big Data Analytics. Massive information processing in transportation systems facing difficulties in tems of data privacy, security, data storage, and data quality. Various Big Transportation data analytics are performed, such as video and audio analytics, image analytics, and historical analytics. The globe is monitored and managed with Web of Devices (WoD) efficiently and effectively.
\end{abstract}

Key words: Big Data, Hadoop, Spark, Flink, Stockpile, WoD.

\section{INTRODUCTION}

In the world, data is generated and integrated from varied sources like social media, geospatial data, and linguistic communication texts. Data could homogeneous or heterogeneous. Some ancient database tools are compatible solely with homogeneous data. We living inside the data age of terabytes or petabytes of data generated from varied businesses, science, engineering, society, medicine, totally different aspects of data pour into computer networks and world data ana lytics has developed from varied domains also as visual analytics, massive data processing, perceptive analytics. Data mining is additionally stated as finding facts from the raw data. Ba sic techniques and ideas of data mining are to get fascinating pattems or knowledge from a great amount of data in varied applications. Data processing may be applied to any quite data a s long because the data is meant for a target a pplication. The quick-growing of massive data has brought data processing doma in a much bigger role moreover as bigger challenges. Massive data is having to be compelled to include every unstructured and semi structured data to boot to the predominated structured data. The bigger challenges of kind of data and a lternative cha llenges including data quantity and the speediness of data production have accumulated hugely within recent years. Data volume, data variety, and data speed measure the 3 main characteristics of massive data. The globe wide data has accumulated from fifty petabytes to two hundred petabytes from 2008 to 2015[1]. Data investigative shows an especially essential part within the smart vehicle framework. Many software applications are developed similarly to disaster intermediary waming regularities, computerized group action of speediness controls, active stoplight a rranging, car to car transmission and cooperation, and actual road traffic forecast and redirecting.

Many of the information methods include enormous information methods as well as information processing, automation, AI, knowledge fusion, and thus the social networks and so on [2]. ITS a ims to supply higher service for motorists and travelers in transport frameworks [3-5]. Transport system will experience analysis of huge information within the following a spects:

Big data analytics is handled with la rge quantities of various as well as sophisticated information produced in transport framework. Big data analytics has resolved 3 difficulties: information stockpiling, information investigation, and information managing. Immense information platforms like Apache Spark and Hadoop exist capable to sort out giant extents of information [6].

i) Analysis of enomous information will enhance transportation framework operation potency.

ii) Analysis of enomous information will enhance transportation framework safety level.

\subsection{Functions of DataAnalytics}

There are four a spects of data a nalytics. They are

- Expla natory (Descriptive) Analytics

- Projective(Predictive) Analytics 
- Investigative(Diagnostic) Analytics

- Prescriptive Analytics

These four aspects a re useful for representing the sequence of steps within the data analytics progress. Descriptive data analytics portray the properties of the data in an exceedingly very target application data set to describe the characteristics of a specific individual. Descriptive analytics discernment into the older and analyzing historical data to produce each quantifiable and qualitative data. Dia gnostic data analytics to work out the frequency with that one thing has discovered within the descriptive data analytics or it is related to one thing else. Diagnostic analytics using many techniques together with data storage and data processing techniques. Diagnostic analytics uses OLAP's drill-up and reverse of drill-up i.e., drill-down operations. Diagnostic analytics is each labour-intensive and explorative. Predictive analytics is employed to form predictions supported current data using numerous applied mathematical and statistical models. Predictive analytics predicts what is possible to happen in the future supported past events. The foremost fashionable projecting paradigms are decision trees and neural networks. Projecting paradigms are created with training information and are probabilistic. Prescriptive analytics provides intelligent recommendations regarding the most popular outcomes. Prescriptive and predictive data analytics gets confusion because of there is excellent overlap between them. Prescriptive analytics address the issues that arise in diagnostic analytics. Prescriptive analytics attracts informative, investigative, and projecting analytics. Prescriptive analytics is enforced using perceptive feature analytics.

\subsection{Analysis of Transportation Data}

Data associated with the transportation collected from a variety of data sources contain rich information. Varied analysis can be presented on the transport of information comprising real-time, historical, predictions, video and images, visual a nalysis [7].

\subsubsection{Analysis of Historical Transportation Data}

Data related to historical transport might be investiga ted to find tendencies and examples to assist create prolonged selections and lead town expansion. For instance, traffic streams, the behaviour of the driver, and the use of road frameworks can now be examined before developing a modem transport framework [8]. Besides, the data from transportation can be examined and utilized by manufacturers to regu late their policies, like free tran sport or traffic signs [9]. Town expansion is capable of even get pleasure on or after information gathered from the common population [10]. This effort explored the transit guide crowdsourced using geolocation data, wherever the customer improved community transport guides them to the web chart. Such as the data permit discovery of important inquire roads, peak hour, and the relevant data of different help for the development of conveyance and routing. This is used to study agglomeration algorithmic rule to user preferences and apply these guiding a lgorithms to constellation to improve optimum transport connections to the community

\subsubsection{Investigation of Actual Transportation Data}

Investigation of actual transportation data represents the capacity process, analyze and diffusing the data being short because the appropriate information is existing in the framework. It will offer the data up and about to the second assessment creators just before permit them in the direction of form a higher business option and faster. Requirements for manual updates drive the need for analysis of time and its expectancy could vary after many seconds towards many minutes. For instance, the present adaptative management techniqueneeds to immediately run the analysis and obtaining results to select whether the indicator temporal order must be modified to adjust to the different traffic state data. Diffusing of data to users in a very significant perhaps even longer pause of intervaldata to generate various additiona lright data and to keep away from infobesity that will confuse users or generating a surprising reaction from users. The break between the supply of data and therefore the ought to refresh the user verifies the acceptable latency investigation that desires the choice of the investigative methods used. The investigation inside the data analysis instance can be vital in the big data space within the transport doma in analysis will be conducted to measure the traffic flow is there to observe the accident or the condition of highway robbery adventure and help emergency vehicles to find the best way [11-13]. Route selection procedure which a nalyses the best expertly shortest path is also found inside the works [14]. Perceiving a situation of traffic stream circumstances and design in real-time will be data accumulated from the exploitation done by the wayside devices, Global Positioning System, smart devices, and connected vehicles.

\subsubsection{Predictive Analytics}

Predictive analysis of using large quantities of raw data, big data frameworks gracefully pleasant feasible for the advancement of prescient models. A prediction model using the process of data, ML (Machine Learning) processing, and to be informed about the data and build predictive of potential traffic activity. For instance, predictions of traffic barrier [15]. This prescient framework utilizes an information mining stage known as KNIME to research climate information and traffic stream information gathered from wayside gadgets in Santander Spa in. By using prescient models, the system will predict the handle to succeed a quarter of an hour. The predictive analysis enables the appropriate organization to a rrange for traffic events a re expected. For instance, when car influx is relied upon because of a significant game, the transportation authority will offer explicit vehicles to desire to the occurrence and urge general society to require transport. When a poor climate is anticipated to cause a few crashes open will be encouraged to be extra cautious while driving. Police could convey an extra workforce in square measures any place wrongdoings are without a doubt to happen all through time. Prescient examination licenses the related 
position to need dynamic and defensive activities to help and improve network wellbeing.

\subsubsection{Analysis of Visual transportation data}

The visual analysis depends upon the visualization of the individual to recognize models of data and significantly different from the renovation then planning information on a form looks. A well- designed visual image will enable customers to obtain facts about the information using slight effort perceptive features. Groups of information which will remain known only so that they become obvious maturity in the chart highly. For instance, the associate function referred to as transport crashes analyser analyses car crashes information in New York town and envisions the information onto a guide [16]. The image displays an extreme perception of the accident a rea and varieties of the corresponding object in the accident (example cycle, truck, van, and car riders) together with different characteristics like time, location, injury/death related reason behind the crash. Customers will check the reasoning behind the rate of impact or varieties of the corresponding object in the crash on the chart and the accident a rea on the map. In the visual analysis common to generate several views that are coordinated to allow customers to be able to complexness knowledge. For example, predicts think about social media and rolling transport information credit in Tokyo presents three views coordinated: Heat Map, Animation Ribbon, and Tweet Bubble [17]. Any reading focus on selected terms of information. Map reading the concentration on time-based dimensions of the data, that is, but is a ctive or not with a rating is more than the amount that remains is the day to day analysis. The Animated Ribbon read utilizing simulation and color-coded tape covered on track to see the number of travelers and the alter of the public on certain routes.

\subsubsection{Analysis of Image and Video Transportation Data}

Analyse video transportation with the picture is rising to observe in transport a nd big data analysis. Ba sic support video analytics acceptance tasks such as the registration code and the class of vehicles powered photographs of transportation [18] move. This capability is often used for social control traffic, as, with increasing the potential for travel document dispensation dashing. The purpose of this plan is to find and break down close failures and to know their roots. The objective of the application with the prescience 0 that means to curtail the traffic morta lities and significant damages to 0 . Information from the video also helps to practice security investigation of data traffic [19]. The process is to analyze close to 473 video data periods of twenty roundabouts in Canada namely Quebec. The investigation utilizes various rules like the chance to crash portability extrapolation and collaborations of vehicles. Such investigation will encourage uncover high accident regions and grant the transportation office to formulate the essential change by decrease the number of impacts inside the zones.

\section{BIG DATA STRATEGIES IN ITS}

$\mathrm{AI}$ is the favoured demonstrating and investigation hypothesis in huge information environments, which makes it ea sy to get examples and paradigms from an enomous amount of information. AI paradigms are regularly ordered into supervised, unsupervised, and reinforcement leaming procedures. Labelled set up information is employed in a regulated discovering algorithmic program. The paradigms employ to enter documents then accordingly an actual result to rea lise the work or guide one of them. Joined a longside the scholarly paradigm and in this way the information record, the concealed yields are regularly anticipated.

The exhibition of regression is to explain the association among single varying and single or additional autonomous factors. Linear regression is that the highly nomally utilized supervised learning. Linear regression is fantastically straightforward, powerful, at ease to decipher, and at ease to program. Notwithstanding its straightforwardness, simple regression is especially lucky in numerous ITS projections, like traffic stream forecast, traffic speed estimate, and transport journey route investigation [20].

A decision tree may be an aftereffect of supporting a device that fills in as a chain of command like a chart to display determinations and their gettable outcomes [21]. Because of their smallness, quality, and straightforwardness, decision trees are broadly used in various ITS projections, like car crash discovery, mishap seriousness examination [22], and travel mode choice. ANN (Artificial Neural Network) may be an innovative ca se of flexible and solid administered lea ming for each characterization and relapse. With enough concealed layers of procedure hubs and preparing data, ANN will get familiar with any non-direct relations among info and target data. As an information displaying device, it has also been embraced in ITS like traffic stream expectation [23], travel stretch forecast, auto collision discovery, and remaining stopping regions forecast.

SVM (Support Vector Machine) is one more familiar supervised learning strategy that utilizations marked information for relapse and a rrangement. Among all the large information investigation model apparatuses in ITS, SVMs have pulled in plea sant premiums in the examination zone. it has been effectively used in movement stretch expectation, the transport time of appearance forecast, and car crash identification. Unsupervised learning typically to boot referred to as agglomeration focus on leaming natural cluster from unlabelled flat information. K-means is that the foremost well likable unsupervised leaming apparatus, and it has been generally received in fundamental street transportation a dvancement, and tra vel span forecast [24].

Reinforcement lea ming expects to curtail the day's end worth through investigation and ga in proficiency with the least complex methodology by communicating with the 
exploratory information. Fortification learning is very pertinent to the board and improvement hypothesis, and it has been assessed to be very strong in light-weight the board in ITS [25-28]. Time series analysis could be a set of applied mathematical methods to paradigm and justify instance relative series of data points. Statistic statement uses a paradigm to get extrapolations forecasts for forthcoming events supported identified past events. Statistic data features a usual sequential collection. This varies from typical data mining/machine learning a pplications, wherever every datum is the a ssociate independent rea lization of the conception to be acquired and therefore the ordering of data points inside a data set does not matter.

Cluster ana ly sis may be a set of applied mathematics ways for grouping data that splits a various cluster into smaller teams of comparable data, whose characteristics of relationships are not notable beforehand. This is often a kind of unsupervised leaming because of training data are not applied [29]. Cla ssification may be a set of methods to spot the classes during which new data points belong, supported a training set containing data facts that have previously classified. These techniques are typically thought about as supervised learning recognitions to the reality of a group of training data [30].

The changepoint analysis could be a powerfully strong and versatile tool that well characterizes changes, determining whether a modification has taken place. It is capable of detective work delicate variations; it characterizes the va riations of detective work by providing confidence intervals and confidence levels. It suits additionally sound for the detection of an outliner. The changepoint analysis is compactable for process historical data particularly once addressing big data [31].

\section{BIG DATA ENVIRONMENT FOR ITS}

Analysis of vast data in ITS is developing with facilita te from innovative big data ecosystem. The enomous information stage uses parallel computing capability and a disseminated document framework to change the speedy information strategy. It is fit for making a feeling of enomous scope framework advancement and furthermore supporting large information. Apache Hadoop is that the favoured free programming framework structure for disseminated techniques and the capacity of a huge measure of informational indexes. Hadoop could be an all-inclusive large information strategy stage; any place various kinds of information techniques or information systematic activities will be disseminated. The appropriated technique manufactures Hadoop to be rea sonable for breaking down the information in ITS like shrewd card information, various electronic ga dgets, online life, GPS information, a nd so on. Apache spark is that the most recent foundation of the open hotspot for a major expanse of informational indexes process that remarkably fits undertakings of AI. Spa rk a dopts a similar Hadoop technology as distributed storage, and it permits user programs to loads into a gathering of group memory, and it questions every now and again. Spark is well-fitting to approaches of AI. The huge information investigation draws near, we tend to present inside the last section region unit AI-based for the most part, and that they will decidedly be acted in each spark and Hadoop sta ges. The large information stage with the information examination approaches running subsequently can assume a colossal job in enomous information investigation in ITS.

\section{BIG DATA APPLICATIONS IN ITS}

\subsection{Analysis of Highway Traffic Crashes}

Substantiation exposes within a globe almost 12 lakhs people are deceased and 500 lakhs huit from vehicle crashes every year [32]. In [33] Bayesia n induction and Random forest are embraced during a period crash forecast model to diminish smash perils, and crash dangers. In [34] Classification And Regression Trees (CART), logistical relapse, and variable adaptational relapse connexions to play out the systematic procedure on mechanized vehicle mishap wound information.

\subsection{Highway Traffic Stream Forecast}

Ideal and exact traffic stream information is fundamental for transportation the board. Investigating a lot of information in ITS has a reward in rush hour gridlock stream expectation [35 $-36]$.

\subsection{Open Transportation Administrations Coordinating}

Open transportation enomous information investigation will encourage to realize transportation riders' excursion designs over the transportation coordinate. The riders' excursion examples will be wont to advise choices to transportation administrators with respect to the planning of the administrations. Beside dissimilar wellsprings of traffic estimation information. In [37] described a way stream essentially dependent on nonlinear improvement paradigm to assess active Origin-Destination request that does not might want explicit powerful connection information. Abuse triangula ted portable records of a lot of mysterious clients. In [38] described an approach to foresee nomal every day Origin-Destination visits.

\subsection{Personal Travel Route Designing}

Considering the information from reasonable telephones and car GPS information, certain transport applications give travelers with genuine traffic information [39], someone else give the most appropriate driving courses with the least period [40]. Large information examination in certain transport applications creates immense financial edges by diminishing excursion time, car influxes, contamination, and ozone depleting substance discharges.

\subsection{Rail Transportation Board and Controller}

With the help of advanced IT technology remodeling the rail transportation systems. They are the most recipient of huge data. Typically, often because of that railing transport methods are typically secured frameworks that do the refined process of huge volumes of data like usual position and speed 
of time of arrival and departure of a train of a station and traveler origin-destination (OD) data. Operators of the rail transportation board are better and rail transportation functioning improved by using big da ta analytics.

\subsection{Forecasting Predictive Models for Travel Time}

Travel times have a pivotal influence on transportation and coordination's. From explorers' perspectives, the information with respect to moving interval assists with downsizing la gs and make better obligation through the higher selection of courses. In coordination's, right moving interval approximation may encourage to downsize transport conveyance costs and to broaden the qua lity help of monetary conveyance by move items inside the ideal period. For traffic chiefs, moving time is a fundamental key to the productivity of traffic framework a ctivity [41].

\subsection{Traffic States of the Clustering}

The most general cluster and classification issues in traffic analysis are traffic state cluster [42] and participant behaviour cluster for cluster formation [43]. The cluster of travel time data tried to find homogenized traffic patterns with the common prediction model to be used. A cluster that could be a technique of promising non-parametric procedure statistics tool permits arbitrarily formed clusters to be exposed.

\subsection{Traffic Routing Problem}

A traffic moving dra wback with localized deciding of vehicle managers in city traffic flow technique was investigated, wherever designing method on behalf of vehicle manager is isolated into 2 phases: key designing for a decision of the best course and plan of action development for passing the present street within the best way. A multi agent system design and the important procedure insights-based techniques for assessment 2 routes in a very random display [44][45] and the nearest route search was developed [46] that are allotted at the strategic drawing board. The models were authorized to genuine information and joined into a traffic territory function; whenever power of the methods was assessed. A disseminated advancement method for the traffic moving way was the idea of in [47].

\subsection{Transport Requirement Modelling}

To establish predict behaviour and represent in travel requirements are necessary at the 3 levels of assessment taking: strategic, examining long infrastructure improvement ways, plan of action in addition to the planning and management; and at last, operational as well as congestion management policies in a shorter time. Transport requirement models are statistical methods that predict long quality requirement supported future projections and current conditions. They need specific analytical capabilities, like the prediction of quality requirement, an altemative of route day, choice of the path itself, and illustration of traffic flow within the road network. These frameworks are constrained to precisely assessing changes in parameters (for example, speed, deferrals, and lines coming about) because of the execution of information procurement forms, vulnerability on suspicions, and model structure.

\subsection{Designing/Directing}

Deciding ideal paths in street systems from an offered sourced to a given objective area is an issue of ten tended to in regular daily existence. There are likewise instruments and applications like strategic organizers and traffic test systems that need to tackle countless such path inquiries. Path organizing methods have advanced rapidly as far as productivity and exactness during the most recent years. One of the two directing calculations proposed to think about the separation (Dijkstra, Bellman-Ford utilizing predefined mea surements) between one hub to one another in a plan. The great procedures broaden these computations with strengthening data and an insatiable methodology to improve its outcomes. Metaheuristic big data in Street Transport and Versatility Exploration strategies have been additionally utilized effectively in the calculation of the briefest way. In any case, these arrangements yield moderate inquiry times when we manage sensible street systems, and this reality hampers their use continuously or intuitively applications. Then, again applying forceful heuristics does not generally give exact outcomes. Street systems present basic properties, e.g., systems are scanty, layered, practically planar, and present progressive structures, that help diverse accelerate methods. These procedures depend on a preprocessing phase where assistant data about the system is gotten and commented on and afterward used to quicken ensuing inquiries. Accelerate systems can be arranged into Objective Coordinated Hunt procedures involving various calculations like geometric.

\section{OPEN CHALLENGES}

The fundamental open difficulties of utilizing the huge information examination in ITS are as per the following.

\subsection{Information Assortment}

Because of the continuous development of vehicles and walkers, data gathered in transportation might be incorrect, deficient, or problematic specifically areas or on specific occasions. For example, not every vehicle is inserted with the procedures expected to give continuous area data, and street traffic information from street devices can be absent. A few potential approaches to deal with handle the competitor is to contribute innovative information assortment progressions and enhance the information assortment ability. Using the head way of IoT, latest sen sor frameworks are made each year, which may encourage improve information assortment and information quality. Also, the choice of information getting robotization to restrict manual information entry is furthermore key to information quality im provement.

\subsection{Information Security}

In the time of enormous information, the most testing and concerning issue is security [48]. Singular assurance may be 
spilled during information transmission, stockpiling, and use [49]. Information accumulated from transportation structures needs to be non-singular information, for instance, vehicle region traffic stream information. Rega rdless, security issues have been concerned since singular information a ssortment by everybody and private regions creates after some time. For instance, the area of people and vehicles are simply collected. On the polluted possibility that this information is not painstakingly made sure about people who take this information would hurt the owner of the information. As such, security protection is something basic for large information utilization in ITS. To prevent unapproved expose of the individual confidential data, govemments should make comprehensive information security regulations, which fuse what information can be dispersed the degree of the information conveying and using the primitive guidelines of information scattering, information openness, and various zones [50]. The transportation workplaces should cautiously control the individual information definition invigorate the organization of information security insistence and use additionally created estimations to improve the information security level.

\subsection{Information Stockpiling}

At present, the information capacity has bounced as of Tera Bytes stage headed for Peta Bytes stage, then the advancement in information stockpiling limit is a long-ways after the information improvement. Particularly in ITS, it will create collection of information from the different sensors consistently. Conventional information stockpiling foundation and database instruments have been not able to adapt to the undeniably huge and complex mass information [51]. In this manner, planning the most sensible information stockpiling engineering has become a major challenge. The primary open distributed storage suppliers, for example, Google and Microsoft keep on improving their administrations with coordinated. Enormous data capacities and multi distributed stora ge and half-breed stockpiling are rising as basic zones for massive information stockpiling. The statistic blasting capacities bear favourable circumstances around numerous types of register serious examination remaining tasks at hand. Likewise, joining insight with capacity is additionally a decent arrangement. Undertakings are searching for keen administration devices that can give incorporated examination inside capacity. This empowers them to direct asset observing and utilize capacity foundation.

\subsection{Data Processing}

Practicality is vital to big data applications in ITS, this application incorporates traffic data pre-processing, traffic state acknowledgment, ongoing traffic control, dynamic course direction, and continuous transport planning. Traffic data, which contains various configurations from different sources must be contrasted and the authentic data at that point prepared inside a brief timeframe [52]. The data preparing framework must have the option to process progressively entangled and progressively extending data, Step by step instructions to ensure the procedure practicality and because of huge with quick information is a major test. Several common massive information frameworks, that manage current information resources, for example, Spark, Samza, Flink, and Kafka Streams and Apache Storm have shown up as of late. Moreover, devoted big data handling systems for ITS have additionally been grown, for example, a stage for ongoing traffic controller and assessing the nomal speediness and the clogged segments of a thruway. These preparing structures give great answers to ongoing da ta handling.

\subsection{Data Opening}

To empower transportation administration clients and Application designers to discover and reuse data successfully, the data should be chronicled and made freely available in great excellence. Information excellence alludes towards the precision fulfilment consistency and unwavering quality [53][54]. Without great information excellence, huge information will be misdirecting dynamic and even produce terrible outcomes. Regardless, start-up information with extraord inary excellence may need period with money. There is an exchange of connecting gap in the lead information rapidly requiring little to no effort and making top-notch data accessible at significant expenses which make gap up great quality data one more enormous test. Viable arrangements incorporate the reception of programmed data catching or potentially the usage of synthetic reasoning to check the data . Also, the transportation divisions ought to have data on the boarding procedure established to gua rantee unblemished and exact data.

\section{CONCLUSION}

Public vehicle systems are basic deteminants of personal satisfaction. Several nations face difficulties in attempts to enrich the idea of their open vehicle frameworks. Creating proficient with manageable strategies is critical to Stable vehicle systems. The Global Transport Council [55] report demonstrated that the development as well as accessibility of huge measure of information in the transport space might prompt innovative strategy significant bits of knowledge and operational upgrades of traffic. Specifically, the development in different types of advances, particularly, the extending digitizing of transport foundation systems could increase assessing expert piece unflinching qua lity and augmentation profitability. Additionally, the accessibility of different sorts of data inside the vehicle space empowers leaders to accumulate and triangulate different types of proof progressively or close to continuous to settle on better choices. The amount of transport-related information is presumably going to augment basically by virtue of turning out to be country urban movement and globalization overall advancement of people and product achieving an extension in the proportion of traffic in enormous urban regions. Vehicles make more information from PDAs and the accompanying 
transponders. Customary techniques for social affairs and examining this information to yield significant encounters will be a test. New kinds of information procuring, associating, and planning are prerequisite to authorize urban regions representatives and municipal coordinators to increment supportive comprehension to help dynamic. Large information and examination of fer various opportunities to light up the headway with respect to viable transportation structures. The gigantic proportion of transportation information accumulated by a major information structure enables different kinds of information examination including recorded persistent farsighted video and visual and picture assessment. The out happens to this information examination can support urban organizing give consistent assistance making the rounds and enhance traffic precautions. Other than associated vehicles extend the unbelievable prospective to drive the variety examination and usage of transport statistics. The troubles identified with the utilization of large information consolidate overseeing varied information sources and obliged correspondence transmission limit directing veracity intrinsic in transportation information using different limit structures and ensuring that insurance and information security are regulated suitably across different frameworks.

\section{REFERENCES}

[1]. Vasant Dhar, "Data Science and Prediction", Communications of the ACM, vol. 56, No. 12, pp.64-73, December 2013.

[2]. Gema Bello-Orgaz, Jason J. Jung, David Camacho, "Social Big Data: Recent Achievements and New Challenges", Information. Fusion, vol. 28, pp. 45-59, 2016.

[3]. S.H. An, B.H. Lee, and D.R. Shin, "A Survey of Intelligent Transportation Systems", International Conference on Computational Intelligence, Communication Systems and Networks, IEEE Computer Society, pp. 332-337, 2011.

[4]. N.E. El Fa ouzi, H. Leung, A. Kurian, "Data Fusion in Intelligent Transportation Systems: Progress and Challenges - A Survey", Information Fusion, vol. 12, pp. 4-10,2011.

[5]. Junping Zhang, Fei-Yue Wang, Kunfeng Wang, Wei-Hua Lin, Xin Xu, and Cheng Chen, "Data-Driven Intelligent Transportation Systems: A survey", IEEE Transactions on Intelligent Transportation Systems, vol. 12, No. 4, pp. 1624-1639, December 2011.

[6]. Matei Zaharia, Mosharaf Chowdhury, Tathagata Das, Ankur Dave, Justin Ma, Murphy M ccauley, Michael J. Franklin, Scott Shenker, and Ion Stoica, "Fast and Interactive Analytics over Hadoop Data with Spark," Networked Systems; login, vol. 37, No. 4, pp. 45-51, August 2012.

[7]. Alex Neilson, Indratmo, Ben Daniel, Stevanus Tjandra. "Systematic Review of the Literature on Big Data in the
Transportation Domain: Concepts and Applications", Big Data Research, vol. 17, pp. 35-44, 2019.

[8]. Cristian Chilipirea, Andreea-Cristina Petre, Loredana-Marsilia Groza, Ciprian Dobre, Florin Pop, "An Integrated Architecture for Future Studies in Data Processing for Smart Cities", Microprocessors Microsystems, vol. 52, pp. 335-342, 2017.

[9]. Davide Tosi, Stefano Marzorati, "Big Data from Cellular Networks: Real Mobility Scenarios for Future Smant Cities", in: Proceedings of the IEEE Second International Conference on Big Data Computing Service and Applications, pp.131-141,2016.

[10].Alexey Golubev, Ilya Chechetkin, Konstantin S. Solnushkin, Natalia Sadovnikova, Danila Parygin, Maxim Shcherbakov, "Strategway: Web Solutions for Building Public Transportation Routes using Big Geodata Analysis", in: Proceedings of the 17th International Conference on Information Integration and Web-Based Applications \& Services, 2015.

[11].Paula Ta-Shma, Adnan Akbar, Guy Gerson-Golan, Guy Hadash, Franco is Carrez, Klaus Moessner, "An Ingestion and Ana lytics Architecture for IoT applied to Sma rt City Use Cases", IEEE Internet of Thing s Journal, vol. 5, No. 2, pp. 765-774, 2018.

[12].Sam Aleyadeh, Sharief M. A. Oteafy, Hossam S. Hassanein, "Scalable Transportation Monitoring using the Smartphone Road Monitoring (SRoM) System", in: Proceedings of the 5th ACM Symposium on Development and Analysis of Intelligent Vehicular Networks and Applications, pp.43-50, 2015.

[13].Qi Shi, Mohamed Abdel-Aty, "Big Data Applications in Real-Time Traffic Operation and Safety Monitoring and Improvement on Urban Expressways", Transportation Research Part C, vol. 58, pp. 380-394, 2015.

[14].Alessandro Attanasi, Edmondo Silvestri, Pietro Meschini, Guido Gentile, "Real World Applications using Para llel Computing Techniques in Dynamic Traffic Assignment and Shortest Path Search", in: Proceedings of the IEEE 18th International Conference on Intelligent Transportation Systems, pp. 1-6, 2015.

[15].Jerome Treboux, Antonio J. Jara, Luc Dufour, Dominique Genoud, "A Predictive Data-Driven Model for Traffic-Jams Forecasting in Smart Santader City-Scale Testbed", in: Proceedings of the IEEE Wireless Communications and Networking Conference (WCNC)-Workshops, pp.64-68, 2015.

[16].Eyad Abdullah, AAhmed Emam, "Traffic Accidents Analyzer using Big Data", in: Proceedings of the International Conference on Computational Science and Computational Intelligence, CSCI,pp.392-397, 2015.

[17].Masahiko Itoh, Daisako Yokoyama, Masashi Toyoda, Yoshimitsu Tomita, Satoshi Kawamura, Masanu Kitsurega wa, "Visual Fusion of Mega -City Big Data: An Application to Traffic and Tweets Data Analysis of Metro Passengers", in: Proceedings of the IEEE International Conference on Big Data, pp.431-440, 2014. 
[18].Wei Yuan, Pan Deng, Tarik Taleb, Jiafu Wan, Chaofan $\mathrm{Bi}$, "An Unlicensed Taxi Identification Model Based on Big Data Analysis", IEEE Transactions on Intelligent Transportation Systems, vol.17, No. 6, pp. 1703-1713, June 2016.

[19].P. St-Aubin, N. Saunier, L. Miranda-Moreno, "Large-Scale Automated Proactive Road Safety Analysis using Video Data", Transportation Research Part C, vol. 58, pp. 363-379, 2015, https://dx .doi .org /10 .1016/j .trc .2015 .04 .007 .

[20].N. Zenina and A. Borisov, "Regression Analysis for Transport Trip Generation Evaluation", Information Technology and Management Science, vol. 16, pp. 89-94, 2013, doi: 10.2478/itms-2013-0014.

[21].J. R. Quinlan, "Induction of Decision Trees", Machine Learning., vol. 1, no. 1,pp. 81-106, 1986.

[22].J. Abellan, G. Lopez, and J. De Ofia, "Analysis of Traffic Accident Severity using Decision Rules via Decision Trees", Expert Systems with Applications, vol. 40, no. 15, pp. 6047-6054, 2013.

[23].E. I. Vlahogianni, M. G. Karlaftis, and J. C. Golias, "Optimized and Meta-Optimized Neural Networks for Short-Tem Traffic Flow Prediction: A Genetic Approach", Transportation Research Part C: Emerging Technologies, vol. 13, no. 3, pp. 211-234, 2005.

[24].R. P. D. Nath, H.-J. Lee, N. K. Chowdhury, and J.-W. Chang, "Modified K-Means Clustering for Travel Time Prediction Based on Historical Traffic Data", in Proceedings of the International Conference on Knowledge-Based Intelligent Information and Engineering Systems, pp. 511-521, 2010.

[25].B. Abdulhai, R. Pringle, and G. J. Karakoulas, "Reinforcement Leaming for True Adaptive Traffic Signal Control", Journal of Transportation Engineering, vol. 129, no. 3, pp. 278-285, 2003.

[26].I. Arel, C. Liu, T. Urbanik, and A. G. Kohls, "Reinforcement Lea ming-Ba sed Multi-Agent Sy stem for Network Traffic Signal Control", IET Intelligent Transport Systems., vol. 4, no. 2, pp. 128-135, 2010.

[27].Ana L. C. Bazzan, "Opportunities for Multiagent Systems and Multiagent Reinforcement Leaming in Traffic Control", Autonomous Agents and Multi-Agent Systems, vol. 18, pp. 342-375, June 2009.

[28].L. Li, Y. Lv, and F.-Y. Wang, "Traffic Signal Timing via Deep Reinforcement Leaming", IEEE/CAA J. Automatic Sinica, vol. 3, no. 3, pp. 247-254, July 2016

[29].J Manyika, M Chui, B Brown, J Bughin, R Dobbs, C Roxburgs, A. H. Byers, "Big Data: The Next Frontier for Innovation, Competition and Productivity", McKinsey Global Institute, May 2011, URL: www.mckinsey.com/.

[30].D. Michie, D. J. Spiegelhalter, and C. C. Taylor, "Machine Leaming. Neural and Statistical Classification ", 1994.

[31].Jie Chen, Arjun K. Gupta, "Parametric Statistical Change Point Analysis: With Applications to Genetics, Medicine, and Finance", Second Edition, Birkhäuser. June 2011.
[32].X. Meng et al., "MLlib: Machine Leaming in Apache Spark", Journal of Machine Learning Research., vol. 17, pp. 1-7,2016.

[33]."Global Status Report on Road Safety", World Health Organization, pp. 1-20, 2018.

[34].Q. Shi, M. Abdel-Aty, "Big Data Applications in Real-Time Traffic Operation and Safety Monitoring and Improvement on Urban Expressways", Transport Research Part C, vol.58, pp. 380-394, 2015.

[35].G. Xiong, F. Zhu, H. Fan, X. Dong, W. Kang, and T. Teng, "Novel ITS Based on Space-Air-Ground Collected Big Data", Big Data and Smart Service Systems, pp. 115-137, 2017

[36].R. Li, C. Jiang, F. Zhu, and X. Chen, "Traffic Flow Data Forecasting Based on Interval Type-2 Fuzzy Sets Theory", IEEE/CAA Journal of Automatic Sinica, vol. 3, no. 2,pp. 141-148, April 2016.

[37].S. Jeon, B. Hong, "Monte Carlo Simulation-based Traffic Speed Forecasting using Historical Big Data", Future Generation Computer Systems, vol. 65, pp. 182-195, 2016.

[38].C.-C. Lu, X. Zhou, and K. Zhang, "Dynamic Origin-Destination Demand Flow Estimation Under Congested Traffic Conditions", Transportation Research Part C, vol. 34,pp. 16-37, 2013

[39].L. Alexander, S. Jiang, M. Murga, and M. C. González, "Origin-Destination Trips by Purpose and Time of Day Inferred from Mobile Phone Data", Transportation Research Part C, vol. 58, pp. 240-250, 2015.

[40].[Online]. Available: http://inrix.com/mobile-apps/

[41].[Online]. Available: https://www.waze.com/.

[42].Hong-En Lin, R. Zito, and M. A. P. Taylor, "A Review of Travel-Time Prediction in Transport and Logistics". Proceedings of the Eastern Asia Society for Transportation Studies, vol. 5, pp. 1433-1448, 2005.

[43].W. Weijemars, and E. van Berkum. "Analyzing Highway Flow Patterns Using Cluster Analysis". Proceedings of the 8th International IEEE Conference on ITS. Vienna, pp. 831-836, June 2005.

[44].J. -G. Lee, J. Han, and K.-Y Whang, "Trajectory Clustering: A Partition-and-Group Framework", Proceedings of ACM SIGMOD International Conference on Management of Data. Beijing, June 2007.

[45].M. Fiosins, J. Fiosina, J. P. Müller, and J. Görmer, "Agent-Based Integrated Decision Making for Autonomous Vehicles in Urban Traffic", Advances in Intelligent and Soft Computing, 2011.

[46].M. Fiosins, J. Fiosina, J. P. Müller, and J. Görmer, "Reconciling Strategic and Tactical Decision Making in Agent-Oriented Simulation of Vehicles in Urban Traffic", Proceedings of 4th International ICST Conference on Simulation Tools and Techniques (SimuTools'2011), pp. 144-151.

[47].J. Fiosina, and M. Fiosins, "Selecting the Shortest Itinerary in a Cloud-based Distributed Mobility Network", Distributed Computing and Artificial 
Intelligence, Advances in Intelligent Systems and Computing (AISC), vol. 217, pp. 103-110, 2013.

[48].M. Fiosins, "Stochastic Decentralized Routing of Unsplittable Vehicle Flows Using Constraint Optimization”, Distributed Computing and Artificial Intelligence. Advances in Intelligent Systems and Computing (AISC), vol.217, pp. 37-44, 2013.

[49].M. Smith, C. Szongott, B. Henne, and G. von Voigt, "Big Data Privacy Issues in Public Social Media," in Proceedings of IEEE International Conference on Digital Ecosystems Technology, pp. 1-6, June. 2012.

[50].Q. Wang, C. Wang, K. Ren, W. Lou, and J. Li, "Enabling Public Auditability and Data Dynamics for Storage Security in Cloud Computing", IEEE Transaction on Parallel and Distributed Systems, vol. 22, no. 5, pp. 847-859, May 2011.

[51].O. Tene and J. Polonetsky, "Big Data for All: Privacy and User Control in the Age of Analytics", Northwestern Journal of Technology and Intellectual Property, vol. 11, no. 5, pp. 240-273, April 2013, Article no. 1.

[52].M. Hilbert and P. Lopez, "The World's Technological Capacity to Store, Communicate, and Compute Information”, Science, vol. 332, no. 60, pp.60-65, April 2011.

[53].M. D. Assuncao, R. N. Calheiros, S. Bianchi, M. A. S. Netto, and R. Buyya, "Big Data Computing and Clouds: Trends and Future Directions", J. Parallel Distrib. Comput., vols. 79-80, pp. 3-15, 2015.

[54].J. Liu, J. Li, W. Li, and J. Wu, "Rethinking Big Data: A Review on the Data Quality and Usage Issues", ISPRS Journal of Photogrammetry Remote Sensing, vol. 115, pp. 134-142, 2016.

[55].“Big Data and Transport: Understanding and Assessing Options", International Transport Forum, OECD/ITF 2015, www. Internationaltransportforum.org. 